\title{
Modelo Experimental para Endometriose em Coelhas com Seǵuimento Evolutivo das Lesões
}

\author{
Experimental Endometriosis Model in Rabbits with Follow-up of the Lesions \\ Julio César Rosa e Silva ${ }^{1}$, Ana Carolina Japur de Sá Rosa e Silva ${ }^{1}$, Pedro Soler Coltro ${ }^{1}$, \\ Sérgio Britto Garcia ${ }^{2}$, Francisco Cândido dos Reis ${ }^{1}$, Antônio Alberto Nogueira ${ }^{1}$
}

\section{RESUM0}

Objetivos: desenvolvimento de modelo experimental de endometriose em coelhas avaliando a evolução temporal da doença macro e microscopicamente.

Métodos: foram utilizadas 30 coelhas nas quais induziu-se lesão de endometriose por fixação de fragmento do corno uterino no peritônio da parede pélvica. Após 4 ou 8 semanas verificou-se a viabilidade da lesão por laparoscopia. Foi documentado o aspecto visual endoscópico e feita avaliação anatomopatológica. Os grupos foram comparados quanto à presença de lesão visual à laparoscopia, seu maior diâmetro, presença de aderências $e$ histologia da mesma. Na análise estatística foram utilizados os testes t de Student e de MannWhitney, com significância estatística de 5\%.

Resultados: em todos os casos foi identificada a presença de lesão visual à laparoscopia após 4 semanas, sendo 64\% císticas, e em $80 \%$ dos casos após 8 semanas, $66 \%$ císticas. As aderências estavam presentes em $71 \%$ das coelhas após 4 semanas (sendo ausentes nos implantes) e em $80 \%$ das coelhas após 8 semanas (13\% nos implantes). O diâmetro das lesões após 8 semanas de implante foi maior que após 4 semanas ( $p<0,0001)$. A análise histológica mostrou $100 \%$ de tecido endometrial (glândula e estroma) em atividade nos 2 grupos.

Conclusão: a utilização desse modelo experimental de endometriose em coelhas mostrou ser possivel reproduzir a doença nesse animal, sendo viável e de fácil execução. Permitiu documentar as características e a progressão dos implantes, o crescimento e o desenvolvimento histopatológico. Embora com mesmo aspecto histológico, as lesões após oito semanas foram maiores que após 4 semanas da implantação.

PALAVRAS-CHAVE: Endometriose. Endometriose: modelo experimental. Laparoscopia.

\section{Introdução}

A endometriose é, atualmente, uma das doenças ginecológicas mais estudadas. Sua incidência é variável de acordo com a população analisa-

\footnotetext{
${ }^{1}$ Departamento de Ginecologia e Obstetrícia da Faculdade de Medicina de Ribeirão Preto - USP

${ }^{2}$ Departamento de Patologia da Faculdade de Medicina de Ribeirão Preto - USP

Correspondência:

Antônio Alberto Nogueira

Departamento de Ginecologia e Obstetrícia

Faculdade de Medicina de Ribeirão Preto - Universidade de São Paulo

Av. Bandeirantes, 3900 - Campus da USP

14049-900 - Ribeirão Preto - SP
}

da, desde 2 a 7,5\% em pacientes assintomáticas submetidas à laqueadura tubária ${ }^{1,2}$ até $50 \%$ nas pacientes com dor pélvica associada à infertilida$\mathrm{de}^{3}$. Caracteriza-se pela presença de tecido endometrial, seja ele glandular ou estromal, fora da cavidade uterina ${ }^{4}$. As lesões de endometriose podem ter aspectos variáveis, podendo ser classificadas em típicas e atípicas. As lesões típicas são as negras e as atípicas as vesiculares, brancas, vermelhas e marrons. Alguns autores defendem a teoria de que estas seriam manifestações evolutivas de doença progressiva ${ }^{5,6}$.

A etiopatogenia da endometriose continua controversa e várias são as teorias atuais para explicá-la, embora nenhuma delas o faça completamente quando isolada. A mais aceita é a teoria 
de Sampson ${ }^{4}$, que aponta a presença de células endometriais viáveis no fluxo menstrual retrógrado como causador das lesões. No entanto, praticamente $90 \%$ das mulheres têm trompas pérvias e apresentam fluxo menstrual retrógrado ${ }^{7}$ e a maioria não apresentará endometriose. Há também a teoria da metaplasia celômica ${ }^{8}$ e, mais recentemente, a teoria imunológica ${ }^{9-11}$. Hoje se acredita na confluência destas teorias para a explicação da etiopatogenia da endometriose. Haveria realmente fluxo menstrual retrógrado associado a predisposição imunológica no microambiente peritoneal que facilitaria o implante de células endometriais viáveis e com potencial para implantação.

A complexidade na sua fisiopatologia e a heterogeneidade da doença em humanos faz da endometriose uma das doenças mais estudadas da atualidade. Para facilitar estes estudos alguns modelos experimentais vêm sendo propostos. O achado de endometriose espontânea em 25\% de animais primatas não humanos ${ }^{12}$ com histologia e localização semelhantes às encontradas em humanos $^{3,13-15}$ levou à utilização destes animais como modelo experimental, porém sua utilização vem sendo progressivamente reduzida devido às dificuldades éticas e pela heterogeneidade das lesões. As ratas seriam modelos experimentais bem mais acessiveis com lesões homogêneas e manejo fácil ${ }^{16}$, mas o pequeno porte do animal dificulta a abordagem cirúrgica com material laparoscópico. Assim, a utilização de coelhas passa a ser boa opção de modelo experimental, pois apresenta baixo índice de infecção, sem necessidade, portanto, de utilização de antibióticos ${ }^{17}$. O porte do animal facilita o acesso por vídeo-laparoscopia se comparado com ratas, e a ausência de ciclo estral dispensa a necessidade de programação dos procedimentos de acordo com a fase do ciclo do animal. Além disso, apresentam lesões homogêneas, geralmente massas sólidas hemorrágicas, facilmente obtidas por meio de autotransplante de fragmentos endometriais ou abertura e exposição da cavidade endometrial ${ }^{18}$. A opção pela técnica cirúrgica de colocação do implante na parede abdominal foi feita pela necessidade de visualização fácil da lesão à laparoscopia.

A padronização e validação de técnica facilmente reprodutivel tornam-se extremamente importantes para o desenvolvimento deste tipo de pesquisa, permitindo simular novas propostas diagnósticas e terapêuticas para a abordagem desta doença. Não encontramos na literatura nenhuma descrição de modelo experimental para endometriose em coelhas com seguimento evolutivo das lesões utilizando-se da técnica laparoscópica e acreditamos ser este um modelo prático, que permitiria caracterizar e documentar as lesões induzidas com baixas taxas de aderências pélvicas por ser método pouco invasivo. A proposta deste estudo é de produzir lesões endometrióticas com o emprego de laparotomia e realizar o seguimento evolutivo da lesão por videolaparoscopia e estudo histológico comparativo das lesões com quatro e oito semanas após o implante.

\section{Materiais e Métodos}

Trata-se de estudo experimental realizado no setor de cirurgia experimental do Hospital das Clínicas do Departamento de Cirurgia e Anatomia e no Departamento de Patologia da Faculdade de Medicina de Ribeirão Preto da Universidade de São Paulo. Foram utilizadas 30 coelhas adultas Nova Zelândia, fêmeas, virgens, com peso aproximado de $2,5 \mathrm{~kg}$. As coelhas foram mantidas em gaiolas apropriadas sob controle de temperatura, umidade e luminosidade do ambiente por três dias antes da indução cirúrgica das lesões, alimentadas com água e ração ad libitum. As coelhas eram pesadas no dia do procedimento inicial.

Todas foram submetidas a laparotomia para indução da lesão de endometriose, após anestesia geral endovenosa com $3 \mathrm{~mL}$ de tionembutal associado a $1 \mathrm{~mL}$ de xilestasina. Procedeu-se à tricotomia da parede abdominal dos animais, seguida dos cuidados de anti-sepsia. As luvas de látex foram lavadas com soro fisiológico $0,9 \%$ exaustivamente para redução da formação de aderências e o mesmo pesquisador realizou todos os procedimentos.

A abertura da cavidade pélvica foi realizada com incisão longitudinal mediana de aproximadamente $2 \mathrm{~cm}$, iniciando $2 \mathrm{~cm}$ cranialmente ao púbis do animal. Posteriormente foi ressecado segmento de cerca de $4 \mathrm{~cm}$ do corno uterino direito, seguido de seu fechamento. A porção uterina ressecada foi imersa em soro fisiológico $0,9 \%$ a $4^{\circ} \mathrm{C}$ por cerca de 2 minutos e então incisada longitudinalmente, sendo retirado fragmento de 5 por $5 \mathrm{~mm}$. Este fragmento de tecido endometrial foi suturado ao peritônio próximo ao trato reprodutivo da coelha, utilizando-se fio Vicryl 6.0, com a face endometrial livre voltada para a cavidade abdominal, com posterior fechamento da incisão cirúrgica abdominal. Nenhuma suplementação hormonal foi administrada antes ou após a laparotomia.

As coelhas foram divididas em dois grupos, sendo o primeiro denominado grupo 8 semanas, constituido de 15 animais, que foram submetidos a laparoscopia diagnóstica para pesquisa de lesões de endometriose, documentação da lesão por meio de vídeo e medida da lesão após oito semanas de sua indução. O outro grupo, chamado grupo 4 semanas, também composto de 15 animais, foi submetido aos mesmos procedimentos porém somente após quatro semanas de seguimento. A 
laparoscopia foi realizada utilizando-se óptica de $6 \mathrm{~mm}$ e $30^{\circ}$ e trocarte de $7 \mathrm{~mm}$. Após incisão longitudinal na pele e tecido subcutâneo de $7,0 \mathrm{~mm}$, sobre a linha mediana e $6,0 \mathrm{~cm}$ abaixo do esterno, foi introduzido de maneira direta um trocarte de 7 mm. Procedeu-se à insuflação de gás $\mathrm{CO}_{2}$ com fluxo constante de um litro por minuto e pressão máxima de aproximadamente $3 \mathrm{mmHg}$ e realizada a inspeção da cavidade abdômino-pélvica, com registro das imagens por meio de vídeo super VHS. Após a inspeção pélvica, identificação e documentação da lesão, as coelhas foram sacrificadas e a lesão foi excisada para análise histológica. Os tecidos retirados foram fixados em formol 10\%, processados para inclusão em parafina e corados por hematoxilina e eosina (HE).

Os grupos foram analisados quanto ao peso médio das coelhas, ganho de peso total ao longo do seguimento e tempo cirúrgico da laparotomia durante indução da lesão endometriótica para homogeneização das amostras e padronização para posterior comparação. A análise estatística utilizou o programa GraphPad Prism ${ }^{\circledR} 2.0132$ Bit Executable (GraphPad Software Inc., San Diego, CA, EUA), utilizando-se o teste estatístico $t$ de Student para as variáveis peso e tempo de cirurgia, que seguiram padrão de distribuição normal sem diferença à análise de variância. O teste de Mann-Whitney foi empregado para a comparação do ganho de peso ao longo de seguimento, pois esta variável não apresentou distribuição gaussiana. Os grupos foram então comparados quanto ao aspecto visual da lesão e tamanho da mesma; para esta comparação foi utilizado o teste de MannWhitney, apesar da distribuição normal, já que os grupos apresentavam variâncias diferentes. Considerou-se o nível de significância de $5 \%$.

Este estudo foi aprovado pela Comissão de Ética em Experimentação Animal da Faculdade de Medicina de Ribeirão Preto - Universidade de São Paulo.

\section{Resultados}

Os grupos apresentaram-se homogêneos quanto ao peso no início do experimento e ganho de peso ao longo do seguimento. O peso inicial foi de $2493,0 \pm 231,4 \mathrm{~g}$ no grupo 8 semanas e de $2453,0 \pm 269,6 \mathrm{~g}$ no grupo 4 semanas, com $\mathrm{p}=0,66$, e o ganho de peso foi de 440,0 $\pm 284,9 \mathrm{~g}$ e de $293,3 \pm 301,1$ g para os grupos 8 e 4 semanas, respectivamente $(\mathrm{p}=0,18)$. O tempo cirúrgico médio da realização da laparotomia para indução da endometriose, no grupo 8 semanas, foi de 18,6 minutos e no grupo 4 semanas, de 16,0 minutos $(p=0,02)$.
Na comparação entre os achados cirúrgicos da videolaparoscopia verificamos que a lesão foi visualizada em $80 \%$ das coelhas do grupo 8 semanas e em $100 \%$ do grupo 4 semanas, embora após o sacrifício do animal e exploração do peritônio via aberta tenham sido encontradas lesões em todos os animais, de ambos os grupos. Das lesões visíveis na laparoscopia, $66 \%$ foram caracterizadas como de aspecto cístico no grupo 8 semanas, semelhante ao achado do grupo 4 semanas, em que este aspecto foi descrito em $64 \%$ das lesões.

As aderências estavam presentes em 71\% das coelhas após 4 semanas, porém em locais distintos dos implantes endometriais, e em $80 \%$ das coelhas após 8 semanas, sendo 13\% no local dos implantes.

$\mathrm{Na}$ avaliação da medida das lesões encontramos diferença significante entre os dois grupos, sendo maiores as lesões após 8 semanas de seguimento. A média do maior diâmetro das lesões do grupo 8 semanas foi de $1,82 \pm 0,73 \mathrm{~cm}$ (variando de 0,7 a $3,5 \mathrm{~cm}$ ) e do grupo 4 semanas, de $0,96 \pm 0,17 \mathrm{~cm}$ (variando de 0,7 a $1,3 \mathrm{~cm})(\mathrm{p}<0,0001)$ (Figura 1).

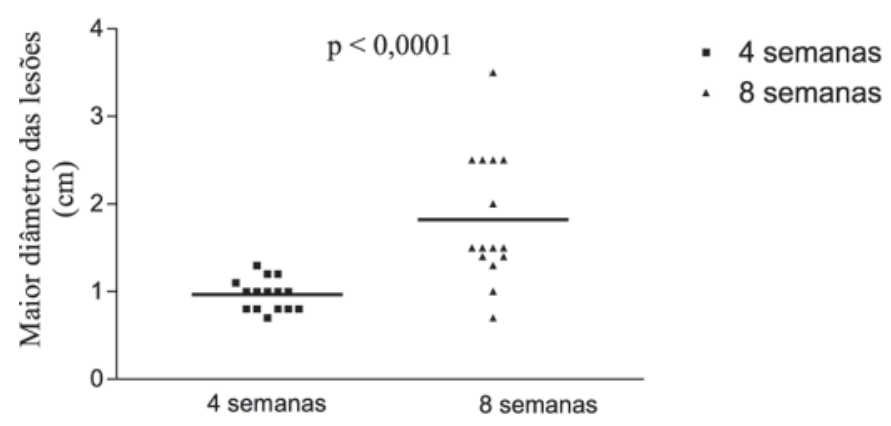

Figura 1 - Distribuição dos diâmetros das lesões das coelhas de acordo com o período de avaliação.

A análise histológica mostrou que todas as amostras eram compostas de tecido endometrial (glândula e estroma) em atividade nos dois grupos, com características morfológicas similares entre eles. As lesões caracterizaram-se por cistos de paredes finas localizados sobre a musculatura estriada da parede abdominal, projetando-se para a cavidade abdominal. A parede destes cistos era formada por camada delgada de tecido conjuntivo, rica em células e revestida por epitélio pavimentoso simples. O estroma era rico em fibroblastos e apresentava alguns macrófagos e eosinófilos, além de grande quantidade de glândulas endometriais típicas. Portanto, o tecido da lesão de endometriose não sofreu alterações em sua composição e arquitetura ao longo do tempo, porém apresentou aumento de suas dimensões. 


\section{Discussão}

O desenvolvimento das lesões após o implante do tecido endometrial ocorreu em todos os animais, com presença de estroma e glândulas à histologia, fato também relatado em outros estudos experimentais. Schor et al. ${ }^{17}$ e Schenken e Asch $^{19}$ descrevem inclusive os implantes como bem vascularizados e bem delimitados, com incremento substancial de tamanho após sete semanas da fixação ao peritônio. Esta facilidade de implantação do tecido endometrial ectópico traduz a alta eficácia deste modelo experimental para desenvolvimento da endometriose, o que talvez permitisse realização do estudo com menor número de animais, apesar de que a demonstração da evolução significativa no diâmetro da lesão não teria sido possivel com casuística menor.

A avaliação videolaparoscópica da lesão, por ser menos invasiva que a laparotomia, possibilita a avaliação e documentação seriada do aspecto macroscópico das lesões e a demonstração de sua evolução temporal. Esta evolução é bastante evidente em nosso estudo, no qual houve incremento significativo no maior diâmetro da lesão entre a $4^{\mathrm{a}}$ e a $8^{\mathrm{a}}$ semana após o implante do tecido endometrial. A progressão do tecido endometrial ectópico sugere viabilidade do implante e provável evolução da doença, e a predominância do aspecto cístico das lesões caracteriza atividade secretora destes focos. Não houve, porém, mudança nas características histológicas do tecido endometrial ectópico quando analisado 4 e 8 semanas após o implante, sem mudança na cor ou aspecto visual das lesões à macroscopia e sem alterações no padrão de distribuição glandular ou estromal à microscopia. Esses dados são concordantes com os da literatura, que não fazem menção a nenhuma mudança na histologia dos implantes e, sim, crescimento das lesões medidas pelo maior diâmetro ou pelo volume.

Um quinto das lesões endometrióticas encontradas após a abertura da cavidade abdominal das coelhas do grupo 8 semanas não haviam sido vistas durante a laparoscopia, pois encontravamse aderidas a estruturas adjacentes. A visualização a céu aberto e a lise destas aderências permitiram identificação de todas as lesões.

O peso das coelhas não diferiu entre os grupos, mostrando, provavelmente, que foram mantidas sob mesmas condições de alimentação, luz e temperatura, não havendo, portanto, interferências das condições ambientais no tamanho das lesões entre eles.

As primeiras coelhas submetidas à indução de lesão de endometriose foram as do grupo 8 semanas, o que talvez justifique o maior tempo ci- rúrgico encontrado nos procedimentos deste grupo em relação ao grupo 4 semanas; não acreditamos, porém, que isto possa ter influenciado na evolução dos implantes.

A utilização dos modelos experimentais para testes terapêuticos com drogas já existentes no mercado, como o danazol, os agonistas do GnRH e inibidores da aromatase, e com novas substâncias ainda em fase de experimentação já vem sendo empregada por alguns autores, com sucesso ${ }^{20-25}$. A verdadeira equivalência destes focos de tecido endometrial implantado na parede de ratas e coelhas com a endometriose humana não é conhecida, por isso estudos comparativos são necessários para que os resultados destes experimentos possam ser extrapolados para humanos.

Com base nos achados deste estudo concluímos que este modelo experimental mostrou-se eficaz no desenvolvimento de focos de endométrio ectópico na parede abdominal de coelhas, mantendo características evolutivas que sugerem progressão da lesão ao longo do tempo e viabilidade do tecido implantado. A possibilidade de realização de laparoscopias ou microlaparoscopias seriadas permite a avaliação macroscópica do aspecto evolutivo da lesão, registro das imagens e coleta de material para exame histológico quando necessário, de maneira minimamente invasiva.

\section{Abstract}

Purpose: development of a new experimental model of endometriosis induction in rabbits evaluating its temporal evolution both macro-and microscopically.

Methods: thirty female rabbits were submitted to endometriosis induction through the fixation of a piece of the left uterine horn to the abdominal peritoneum. After four or eight weeks the viability of the lesions was verified by laparoscopy. The lesions were observed endoscopically. The implants were measured and histological analyses were made. The groups were compared for the presence of endometriotic lesion on laparoscopy, presence of adhesions, implant size and histological aspects. For statistical analyses we utilized Student's t and Mann-Whitney's tests, with a statistical significance of 5\%.

Results: endometriotic lesions were identified in all cases submitted to laparoscopy after 4 weeks of induction, $64 \%$ of them cystic, and in $80 \%$ of the rabbits after eight weeks, $66 \%$ of which cystic. The adhesions were present in $71 \%$ of the rabbits after 4 weeks (none in the implants) and in $80 \%$ of the rabbits after 8 weeks (13\% in the implants). The lesions were significantly larger after 8 weeks $(p<0,0001)$. The histological analyses showed 100\% of endometrial tissue in both groups.

Conclusion: this experimental model showed that it is possible to simulate endometriosis in rabbits with a viable and simple technique, also allowing to record the characteristics and development of the implants macro-and microscopically. 
Although the histological aspects were similar, the lesions after eight weeks were larger than after four, making their manipulation easier.

KEYWORDS: Endometriosis. Endometriosis: experimental model. Laparoscopy.

\section{Referências}

1. Strathy JH, Molgaard CA, Coulam CB, Melton LJ 3rd. Endometriosis and infertility: a laparoscopic study of endometriosis among fertile and infertile women. Fertil Steril 1982; 38:667-72.

2. Kirshon B, Poindexter AN 3rd, Fast J. Endometriosis in multiparous women. J Reprod Med 1989; 34:215-7.

3. Cornillie FJ. Peritoneal endometriosis. In: Brosens I, Gordon E, editors. Tubal infertility. $1^{\text {st }}$ ed. London: Gower Medical; 1990. p. 3-8.

4. Sampson JA. Peritonial endometriosis due to menstrual dissemination of endometrial tissue into the peritonial cavity. Am J Obstet Gynecol 1927; 14:422-69.

5. Redwine DB. The distribution of endometriosis in the pelvis by age groups and fertility. Fertil Steril 1987; 47:173-5.

6. Koninckx PR, Meuleman C, Demeyere S, Lesaffre E, Cornillie FJ. Suggestive evidence that pelvic endometriosis is a progressive disease, whereas deeply infiltrating endometriosis is associated with pelvic pain. Fertil Steril 1991; 55:759-65.

7. Halme J, Hammond MG, Hulka JF, Raj SG, Talbert LM. Retrograde menstruation in healthy women and in patients with endometriosis. Obstet Gynecol 1984; 64:151-4.

8. Suginami H. A reappraisal of the coelomic metaplasia theory reviewing endometriosis occurring in unusual sites and instances. Am J Obstet Gynecol 1991; 165:214-8.

9. Chung HW, Lee JY, Moon HS, et al. Matrix metalloproteinase-2, membranous type 1 matrix metalloproteinase, and tissue inhibitor of metalloproteinase-2 expression in ectopic and eutopic endometrium. Fertil Steril 2002; 78:787-95.

10.Braun DP, Ding J, Shen J, Rana N, Fernandez BB, Dmowski WP. Relationship between apoptosis and the number of macrophages in eutopic endometrium from women with and without endometriosis. Fertil Steril 2002; 78:830-5.

11.Witz CA, Cho S, Montoya-Rodriguez IA, Schenken $\mathrm{RS}$. The a $2 \mathrm{bl}$ and $\mathrm{a} 3 \mathrm{bl}$ integrins do not mediate attachment of endometrial cells to peritoneal mesothelium. Fertil Steril 2002; 78:796-803.

12.D'Hooghe TM, Bambra CS, Cornillie FJ, Isahakia M, Koninckx PR. The prevalence and laparoscopic appearance of spontaneous endometriosis in the baboon (Papio anubis, Papio cynocephalus). Biol Reprod 1991; 45:411-6.

13.Cornillie FJ, Lauweryns JM, Brosens IA. Normal human endometrium. An ultrastructural survey. Gynecol Obstet Invest 1985; 20:113-29.

14. Clement PB. Pathology of endometriosis. Pathol Annu 1990; 25:245-95.

15.Vernon MW, Wilson EA. Studies on the surgical induction of endometriosis in the rat. Fertil Steril 1985; 44:684-94.

16. Berthet J, Pasquier D, Racinet C. An original model of experimental endometriosis in the rabbit. $\mathrm{J}$ Gynecol Obstet Biol Reprod (Paris) 1992; 21:625-8.

17.Schor E, Freitas V, Soares Júnior JM, Simões MJ, Baracat EC. Endometriose: modelo experimental em ratas. Rev Bras Ginecol Obstet 1999; 21:281-4.

18. Manyak MJ, Nelson LM, Solomon D, DeGraff W, Stillman RJ, Russo A. Fluorescent detection of rabbit endometrial implants resulting from monodispersed viable cell suspensions. Fertil Steril 1990; 54:356-9.

19.Schenken RS, Asch RH. Surgical induction of endometriosis in the rabbit: effects on fertility and concentrations of peritoneal fluid prostaglandins. Fertil Steril 1980; 34:581-7.

20.Hahn DW, Carraher RP, Foldesy RG, McGuire JL. Development of an animal model for quantitatively evaluating effects of drugs on endometriosis. Fertil Steril 1985; 44:410-5.

21.Jones RC. The effect of a luteinizing hormone releasing hormone (LRH) agonist (WY-40, 972), levonorgestrel, danazol and ovariectomy on experimental endometriosis in the rat. Acta Endocrinol (Copenh) 1984; 106:282-8.

22.Sakata M, Terakawa N, Mizutani T, et al. Effects of danazol, gonadotropin releasing hormone agonist, and a combination of danazol and gonadotropinreleasing hormone agonist on experimental endometriosis. Am J Obstet Gynecol 1990; 163:1679-84.

23.Zanagnolo VL, Beck R, Schlaff WD, Damewood MD, Bobbie D, Rock JA. Time-related effects of a gonadotropin releasing hormone analog treatment in experimentally induced endometriosis in the rat. Fertil Steril 1991; 55:411-5.

24.Tjaden B, Galetto D, Woodruff JW, Rock JA. Timerelated effects of RU486 treatment in experimentally induced endometriosis in the rat. Fertil Steril 1993; 59:437-40.

25.Zanella SR, Tsuruda RH, Guzzoni SR, Trautwein VW, Beck RT. Endometriose: tratamento com agonista do GnRH a partir de lesão induzida em ratas. Rev Bras Ginecol Obstet 1994; 16:125-8.

Recebido em: 16/8/04

Aceito com modificações em: 16/9/04 